\title{
Francisco José Francés García, Antonio Alaminos Chica, Clemente Penalva Verdú y Oscar Antonio SANTACREU FERNÁNDEZ \\ EL PROCESO DE MEDICIÓN DE LA REALIDAD SOCIAL: LA INVESTIGACIÓN A TRAVÉS DE ENCUESTAS \\ Cuenca (Ec): PYDLOS Ediciones, 2014
}

\author{
Carlos Mora Fernández \\ Universidad de Alicante (Spain) \\ carlosmora432@gmail.com
}

En 1993 nace el Programa de Población y Desarrollo Local Sustentable (PYDLOS), en la Universidad de Cuenca (Ecuador), como respuesta a una serie de acontecimientos sociales y ambientales. Su naturaleza investigadora y aplicada le exige una planificación sistemática sobre el entorno en el que se lleva a cabo su trabajo, por lo que en 2014 pasa a ser Grupo de Investigación del "Departamento Espacio y Población". Esta contextualización nos sirve para comprender la edición por parte de PYDLOS del libro El proceso de medición de la realidad social: la investigación a través de encuestas, en el marco de la conveniencia de contar con un material aplicado para investigadores y docentes para el diseño y desarrollo de proyectos y estudios.

El proceso de medición de la realidad social: la investigación a través de encuestas forma parte de una colección de libros metodológicos en los que además de éste hasta el momento se incluyen también Introducción a los modelos estructurales en investigación social, La investigación cualitativa: técnicas de investigación y análisis con Atlas.ti, La investigación participativa: métodos y técnicas, Introducción a los modelos estructurales en investigación social y Análisis multivariante para las Ciencias Sociales I. Índices de distancia, conglomerados y análisis 
factorial; elaborados todos ellos por los profesores de la Universidad de Alicante Francisco José Francés García, Antonio Alaminos Chica, Clemente Penalva Verdú y Oscar Santacreu Fernández. Con la publicación del último volumen, se pretende cerrar un círculo materializando en sus páginas los procesos necesarios para llevar a cabo una investigación desde cualquier perspectiva metodológica. El interés de esta colección para los investigadores no sólo radica en su excelente explicación de las metodologías cualitativas y cuantitativas, sino que nos ofrece una visión alternativa de otros procedimientos de investigación como puede ser la Investigación Participativa, excluida en numerosas ocasiones de los manuales al uso de técnicas de investigación.

El presente libro coge el relevo de textos de referencia en las ciencias sociales aplicadas como Metodología y técnicas de investigación social de Piergiorgio Corbetta (2007), El análisis de la realidad en la intervención social: métodos y técnicas de investigación de María José Rubio y Jesús Varas (1997) o Metodología cuantitativa, de Cea D'Ancona (1998) y se alza como una obra sintetizada y práctica.

El libro se estructura en dos partes. La primera parte, El proceso de la investigación social nos introduce en las distintas fases que ha de incluir en su trabajo el investigador para llegar al conocimiento de los fenómenos sociales. La capacidad de transmitir a través de ejemplos cotidianos, el uso de un vocabulario comprensible y una estructura que abarca todos los aspectos para la elaboración de un trabajo empírico, ayudará al lector a interiorizar de forma amena los entresijos de este proceso que puede parecer complejo a los que se inician en el campo de la investigación social. El conocimiento de la realidad social, como primer capítulo, es referente de la idea expuesta sobre la naturaleza del material que tenemos entre las manos, por ejemplo, la lógica de la investigación o el rol del investigador como objeto y sujeto de estudio, que son explicadas de un manera muy ilustrativa. Tras esta presentación, en el segundo capítulo denominado La investigación social se aborda las cuestiones relativas a las fases previas de la medición social y la importancia que tiene definir correctamente los objetos de estudio, para más tarde profundizar en aspectos como el diseño metodológico o el trabajo de campo. Como es sabido, la acotación geográfico-temporal, la viabilidad de la investigación, la planificación del mismo, así como la correcta redacción de los objetivos, son condición sine qua non para la consecución de las metas planteadas. Llegados a este punto, se comienza a describir las primeras diferencias entre las metodologías cualitativas y cuantitativas, introduciéndonos en la operacionalización de las hipótesis en variables que más tarden nos servirán para su medición. En este reco- 
rrido, nos volvemos a encontrar con recursos que orientan a una interiorización correcta de los conceptos tratados, a través de gráficos, tablas e ilustraciones, donde los autores sintetizan las ideas más complejas. Al llegar a las perspectivas de la investigación social en el tercer capítulo (Niveles y perspectivas en investigación social), se sumerge al lector en uno de los campos fundamentales de la investigación sociológica aplicada, que es el cómo nos acercamos al conocimiento y la relevancia de elegir cuidadosamente los métodos y técnicas acordes a los objetivos del estudio. Estas cuestiones se resuelven en el texto a través de una visión transversal de las perspectivas de investigación utilizando como referentes principales los textos clásicos de Jesús Ibáñez, añadiendo aportaciones más contemporáneas.

La segunda parte, titulada como "La medición de la realidad social: Encuestas y cuestionarios", comprende la esencia del libro que tenemos entre manos. La investigación cuantitativa, que tiene como principal herramienta de investigación social la encuesta, ocupa el grueso de la obra, y de manera coherente y secuencial, van apareciendo los distintos procesos que nos llevan desde la operacionalización hasta el análisis de resultados. En el primer capítulo de esta segunda parte, comienza con la estadística y la encuesta como método. La definición del método y sus características como una de las herramientas más utilizadas en la sociología nos abre camino para ir conociendo poco a poco las principales fases de la investigación con este método. La popularidad de la encuesta tanto en el mundo científico-social, como en los medios de comunicación, infiere a este método la necesidad de conocer sus particularidades. Por otra parte, la operacionalización de conceptos como las variables latentes, requieren de un esfuerzo metodológico que el científico social debe dominar. En el segundo capítulo, nos encontramos con una de las piedras angulares de la encuesta y que, para muchos, es el ámbito más complejo: la operacionalización. Los autores, recorren este concepto con mucho cuidado dando preponderancia a la creatividad sociológica del investigador para trasladar los conceptos teóricos de la realidad social hacia conceptos operativos susceptibles de medición. El paso siguiente tras exponer estas ideas, se encuentra sin lugar a dudas en la comprensión de los conceptos de validez y fiabilidad, que se hacen necesarios su incorporación en la encuesta. Entendiendo por validez la relación entre el concepto teórico y los indicadores con el que lo definimos; por otro lado, la fiabilidad es la capacidad que nos otorga el instrumento de obtener los mismos resultados en distintas pruebas. Atendiendo a estas premisas, el capítulo sigue su itinerario hacia el enfrentamiento de posibles obstáculos metodológicos inherentes a la encuesta, como es el diseño del cuestionario, a la 
aplicación del mismo o el tipo de población al que va dirigido. Al poder encontrarnos en estudios en los que tengamos que hacer inferencias de una población determinada, en el tercer capítulo nos introduce en los diseños muestrales, los tipos de muestra, sus criterios y la importancia de la seleccionar procedimientos óptimos según el objeto de estudio. El detalle sobre cada procedimiento otorga un protagonismo a este capítulo debido a la importancia que tiene diseñar adecuadamente la muestra en un método como es la encuesta.

La entrevista estandarizada y el diseño del cuestionario, sin lugar a dudas ocupan un espacio privilegiado en el texto puesto que muchas controversias metodológicas se ciernen en este proceso. Los autores parecen conscientes de este aspecto y se centran en la estructura que debe tener el cuestionario y el tipo de preguntas teniendo en cuenta que el proceso por el cual se traducen las hipótesis empíricas a la observación se hace necesario acudir a una herramienta que nos permita recoger de la forma más objetiva posibles los datos que nos ofrece la realidad social. Del diseño del cuestionario pasamos a la aplicación del mismo, momento en el que se puede producir un mayor número de sesgos y en el que se lleva a cabo el contacto con la fuente emergente de información. Pero además, los autores nos detallan los distintos tipos de formas que se puede aplicar, desde los tradicionales cara a cara, hasta las oportunidades que nos ofrecen las nuevas tecnologías. En el penúltimo capítulo, relativo al trabajo de campo, se acentúa los procesos de selección y formación de los encuestadores. Por último, se finaliza el libro con la introducción al análisis de resultados, dando así pistas de la publicación del siguiente volumen que versará sobre distintas opciones de análisis de datos.

A modo de conclusión, podríamos afirmas que los autores de El proceso de medición de la realidad social: la investigación a través de encuestas pretenden proporcionar un material didáctico y esquematizado a todos aquellos investigadores e investigadoras que se inician en la investigación social. Conscientes de la relevancia que está adquiriendo el conocimiento de los fenómenos sociales, se hace necesario este conjunto de publicaciones que complementan al resto de la misma temática. Por último, si tuviésemos que destacar algún aspecto del libro, convendría citar el orden coherente expuesto de cada capítulo, las ilustraciones y esquemas que sintetizan cada concepto y la forma de explicar los aspectos más complejos con ejemplos cotidianos que hacen más fácil su comprensión. Además, si tenemos en cuenta el motivo de su elaboración, consistente en suministrar un material útil para aquellos que se introducen en la investigación social, valoraremos que junto al resto de publicaciones, nos encontramos ante una colección interesante y actualizada que aborda las múltiples opciones investigadoras y su práctica. 


\section{BIBLIOGRAFÍA}

Cea D’Ancona, M. (1998) Metodología cuantitativa. Madrid: Síntesis.

Corbetta, Piergiorgio. (2007) Metodología y técnicas de investigación social. Madrid: Mc Graw Hill.

Rubio, M. y Varas J. (1997) El análisis en la intervención social: métodos y técnicas de investigación. Madrid: Editorial CCS.

CARLOS MORA FERNÁNDEZ (Alicante, 1984) es graduado en Sociología por la Universidad de Alicante y técnico superior en Comercio y Marketing. En el año 2016 disfrutó de Beca de Colaboración del Ministerio de Educación, Cultura y Deporte en el Departamento de Sociología II. 